Revue

Revue de l'histoire des religions

de Ihistoire des religions

1 | 2016

Varia

\title{
L'apocalyptique, entre théologie politique et gouvernementalité
}

Apocalyptics, between Political Theology and Governmentality

Elettra Stimilli

(2) OpenEdition

Journals

Édition électronique

URL : https://journals.openedition.org/rhr/8490

DOI : $10.4000 /$ rhr.8490

ISSN : 2105-2573

Éditeur

Armand Colin

Édition imprimée

Date de publication : 1 mars 2016

Pagination : 83-100

ISBN : 978-2-200-93059-2

ISSN : 0035-1423

Référence électronique

Elettra Stimilli, «L'apocalyptique, entre théologie politique et gouvernementalité », Revue de l'histoire des religions [En ligne], 1 | 2016, mis en ligne le 01 mars 2019, consulté le 21 septembre 2021. URL http://journals.openedition.org/rhr/8490 ; DOI : https://doi.org/10.4000/rhr.8490 


\section{L'apocalyptique, entre théologie politique et gouvernementalité}

L'apocalyptique est une catégorie d'interprétation utilisée dans le débat $d u x^{e}$ siècle sur le sens de la politique et de l'histoire après la Shoa, le totalitarisme et la domination planétaire du marché mondial. Le rôle de la religion chrétienne dans le développement de la politique occidentale est au centre de ce débat - depuis la théologie politique de Schmitt et Taubes jusqu'à la philosophie de l'histoire de Löwith et Kojève et au "pouvoir pastoral» et "gouvernemental» de Foucault. Mais l'intérêt évident des premiers auteurs pour l'apocalyptique s'oppose à la présence limitée de cette question dans les études de Foucault. L'objectif de cet article est de comprendre ces différentes positions, en essayant de définir la pertinence du christianisme dans une analyse de l'époque actuelle.

\section{Apocalyptics, between Political Theology and Governmentality}

Apocalyptics is an interpretative category used in the early $20^{\text {th }} \mathrm{C}$ in the debate surrounding the meaning of history and politics following the Shoah, totalitarianism and the planetary domination of the world market. The role of the Christian religion in the development of Western politics was at the core of this debate - which ranged from the political theology of Schmitt and Taubes to the philosophy of history according to Löwith and Kojève and to Foucault's "pastoral power" and "governmental power". However, the obvious interest of the first of these authors in apocalyptics contrasts with the limited presence of the issue in Foucault's studies. The object of this article is to understand these different positions by attempting to define the pertinence of Christianity within an analysis of the current age. 
Au-delà du sens spécifique que le terme «apocalypse» a acquis dans les études d'histoire des religions, le domaine de l'apocalyptique est apparu à l'intérieur de la discussion philosophique du siècle dernier comme un problème politique, surtout au moment où est née la nécessité de trouver une explication à certaines apocalypses historiques (comme la Shoa ou l'utilisation de la bombe atomique). Dans ce contexte, c'est même le sens de la religion chrétienne dans le développement historique de la modernité qui est en jeu. Bien qu'aujourd'hui nombre des prémisses de ce débat aient changé, la discussion sur ce thème est encore présente. À la suite de la suprématie du marché mondial et de sa crise actuelle, la possibilité d'une nouvelle apocalypse économique et écologique est en effet de nouveau annoncée. Reconstruire certaines lignes de ce passage, de l'interprétation du $\mathrm{xx}^{\mathrm{e}}$ siècle sur la modernité jusqu'aux enquêtes plus récentes sur ces questions, permet d'aborder d'une façon différente ce qui pose problème dans le monde actuel.

\section{Fin dE L'HISTOIRE ET KATECHON}

En 1950, Carl Schmitt a publié dans la revue Universitas une recension du livre de Karl Löwith, Histoire et Salut (Meaning in History), publié un an plus tôt à Chicago ${ }^{1}$. Histoire et Salut est l'un des textes de référence du débat du $\mathrm{Xx}^{\mathrm{e}}$ siècle autour du rapport entre apocalypse et modernité. On ne manquera pas, bien sûr, de remarquer que, peu après la sortie du livre de Löwith l'un des plus brillants intellectuels exilés d'origine juive, issu de la sphère intellectuelle heideggerienne -, c'est précisément Carl Schmitt, juriste et théoricien du nazisme, qui décide d'en écrire une recension presque enthousiaste. Mais ce qui saute surtout aux yeux, c'est à quel point l'analyse du travail de Löwith sert, en réalité, de

1. Carl Schmitt, «Drei Stufen historischer Sinngebung», Universitas, 8, 1950, p. 927-931. Karl Löwith, Meaning in History. The Theological Implications of the Philosophy of History, Chicago, The University of Chicago Press, 1949; Histoire et Salut. Les présupposés théologiques de la philosophie de l'histoire, trad. Marie-Christine Challiol-Gillet, Sylvie Hurstel et Jean-François Kervégan, Paris, Gallimard, 2002. 
prétexte à Schmitt pour se confronter à la conception chrétienne de l'histoire. Il en ressort l'idée du katechon, une des catégories centrales de la théorie politique schmittienne des années $1950^{2}$. Bien qu'il s'agisse d'un texte mineur, quelques-unes des questions clés du rapport entre apocalyptique et modernité, débattues dans ces années-là et traitées avec une attention particulière par Löwith, sont plus ou moins explicitement présentes dans cette recension.

Tout le livre de Löwith est destiné à vérifier, dans l'histoire de l'Occident, les différentes manifestations de l'exigence d'accomplissement, propre à la perspective apocalyptique de l'histoire judéo-chrétienne, sa façon d'être tendue vers un eschaton. Dès les premières pages, Löwith cherche à motiver l' " ordre de succession inversé» de son analyse qui, depuis Burckhardt, Marx, Hegel, Vico, Joachim de Flore, Augustin et jusqu'à la conception biblique, «va déployer le processus des interprétations de l'histoire en partant de la fin», et qui pourrait «susciter à première vue de l'étonnement $\aleph^{3}$. Aux yeux de Löwith, cela se justifie parce qu'«[i]l est plus facile de comprendre la pensée d'alors et la foi en la Providence à travers une analyse critique des présuppositions théologiques de la croyance séculière au progrès, que de procéder à l'inverse en faisant découler la croyance moderne au progrès de la théologie de l'histoire antécédente ${ }^{4}$. D'autant qu'à partir de la catastrophe récente - Löwith écrit en 1949 - les difficultés de la philosophie de l'histoire moderne et ses implications théologiques sont plus évidentes. C'est dans ce sens que cette étude se présente comme une réponse aux problèmes fondamentaux apparus avec De Hegel à Nietzsche (Von Hegel bis Nietzche), publié en $1941^{5}$. L'intention de Löwith est de démasquer l'«illusion» implicite dans la philosophie de l'histoire moderne. En fait la philosophie de l'histoire moderne a tendance à

désigner l'interprétation systématique de l'histoire universelle selon un principe directeur qui permet de mettre en relation événements et conséquences historiques et de les rapporter à un sens ultime.

2. Mais Schmitt parle pour la première fois du katechon en 1932: voir C. Schmitt, Glossarium, Aufzeichnungen der Jahre 1947-1951, Berlin, Dunker \& Humblot, 1991, p. 63.

3. K. Löwith, Histoire et salut, p. 22.

4. Ibid., p. 22-23.

5. K. Löwith, Von Hegel bis Nietzsche, Zürich, Europa Verlag, 1941; De Hegel à Nietzsche, trad. Rémi Laureillard, Paris, Gallimard, 1969. 
Ainsi comprise, toute la philosophie de l'histoire devient totalement dépendante de la théologie, c'est-à-dire de l'interprétation théologique de l'histoire comme histoire du Salut. ${ }^{6}$

C'est pour cela que la philosophie de l'histoire est une «illusion» et non pas une «science». Du reste, «comment pourrait-on justifier scientifiquement la foi dans le Salut?» $\gg^{7}$. Le livre de Löwith naît de l'exigence de démontrer comment la philosophie de l'histoire moderne trouve son origine dans la conception eschatologicoapocalyptique de l'histoire, dans «la croyance biblique en la rédemption», et comment elle finit par perdre totalement son sens avec «la sécularisation de son modèle eschatologique ${ }^{8}$. Le fait de démasquer une utilisation erronée des mots «sens» et «but» ou «sens» et «fin», implicite dans l'histoire biblique et portée à ses extrêmes conséquences par le processus moderne de sécularisation, est fondamental pour comprendre ce passage. Ici, le problème est que les termes sont devenus interchangeables, si bien que «la notion de fin [...] détermine la notion de "sens" ${ }^{9}$. «Les événements historiques eux aussi n'acquièrent un sens que s'ils renvoient à une fin située au-delà des événements factuels et, parce que l'histoire est un mouvement dans le temps, cette fin doit être un but à venir $\gg^{10}$, qui rende significatif le processus universel et qui fasse de chaque événement partial un événement «total» et donc totalement compréhensible. Sur la base des prémisses théologiques de la philosophie de l'histoire moderne, le futur, selon Löwith, devient alors le vrai sens de l'histoire, au sens où

[l']importance de cette visée d'une fin ultime, fin au sens de finis et de telos, tient au fait qu'elle met à notre disposition le schéma d'un ordre et d'un sens en progrès qui était en mesure de surmonter l'antique crainte du fatum et de la fortuna. L'eschaton ne se contente pas de mettre un terme au cours de l'histoire, il l'organise et l'accomplit selon un but précis. La pensée eschatologique a le pouvoir de maîtriser la temporalité du temps, un temps qui engloutit ses propres créatures s'il n'est pas délimité de manière sensée par un but ultime. [...] Ce n'est qu'à l'intérieur de cette délimitation eschatologique du progrès historique que l'histoire a pu devenir «universelle». ${ }^{11}$

6. K. Löwith, Histoire et Salut, p. 21 (traduction modifiée).

7. Ibid. (trad. mod.)

8. Ibid., p. 22.

9. Ibid., p. 26.

10. Ibid.

11. Ibid., p. 41. 
Dans sa critique du livre de Löwith, Schmitt admet l'importance de l'analogie entre le $\mathrm{xx}^{\mathrm{e}}$ siècle et la perspective eschatologicoapocalyptique chrétienne. En négligeant le parallèle entre Hitler et Staline, présent au contraire dans Histoire et Salut ${ }^{12}$, et en focalisant plutôt l'attention sur la comparaison entre la dialectique hégéliano-marxisto-stalinienne et la vision chrétienne de l'histoire, Schmitt, avec des accents très élogieux, soutient qu' "aucun auteur n'est aujourd'hui en mesure de nous instruire» sur la situation actuelle «avec autant de clarté du point de vue de la philosophie de l'histoire et avec une aussi grande lucidité intellectuelle que Karl Löwith ${ }^{13}$. Ce qui importe le plus à Schmitt réside dans la solution qu'offre Löwith pour interpréter «la foi des masses» dans la "planification», qui caractérise selon lui le $\mathrm{xx}^{\mathrm{e}}$ siècle. La perspective de la philosophie de l'histoire est essentielle. L'allusion à la division du monde en deux blocs opposés et la référence à la menace de l'utilisation des armes atomiques en tant qu' apocalypse annoncée à l'époque de la «guerre froide» sont présentes dans ce passage initial:

Tous les hommes qui planifient et cherchent à attirer les masses derrière leurs planifications, poussent à une forme ou une autre de philosophie de l'histoire. Ils acceptent le fait que la science moderne de la nature mette entre les mains des détenteurs de pouvoir des moyens d'extermination. Mais la question de savoir contre quels hommes ces moyens seront judicieusement employés n'est pas, de toute évidence, une question qui relève des sciences de la nature. Elle n'est plus, non plus, depuis longtemps, une question relevant de la morale ou du droit. La question et sa réponse ne procèdent plus aujourd'hui que de la philosophie de l'histoire. Le marxisme en particulier est si intensément une philosophie d'histoire, que le moindre contact avec lui se meut en controverse de philosophie de l'histoire. Les élites planificatrices et dirigeantes s'auto-construisent, comme elles construisent les masses qu'elles dirigent, au moyen d'interprétations qui les inscrivent dans la philosophie de l'histoire. Toute propagande de masse cherche son évidence dans la preuve de son inscription du côté des choses à venir. Toute la foi des masses n'est que la foi dans le fait d'être du bon côté, l'adversaire se trouvant du mauvais puisque le temps, l'avenir et le cours des choses travaillent contre lui. Et le désespoir lui-même ne trouve sa dernière énergie que dans la menace qu'est censée faire peser la perte de sens sur l'histoire universelle. ${ }^{14}$

12. Voir ibid., p. 209.

13. C. Schmitt, «Drei Stufen historischer Sinngebung», p. 928.

14. Ibid., p. 927. 
Schmitt partage avec Löwith l'idée que la foi dans le progrès, qui caractérise l'époque moderne, n'est qu'une sécularisation de la vision apocalyptique de l'histoire du judaïsme et du christianisme, puisque c'est «de là qu'elle a tiré ses "eschata" ${ }^{15}$. Toutefois, il pense en même temps que la vision apocalyptique d'une fin des temps, en tant que «temps épuisé», est en nette opposition avec le concept moderne de planification. En effet, «la vive attente d'une fin imminente paraît priver toute histoire de son sens et provoquer une paralysie eschatologique $»^{16}$.

À cette paralysie eschatologique de l'époque moderne Schmitt oppose l'image du katechon, qui est présente dans la conception chrétienne de l'histoire et qui remonte à la deuxième Épître aux Thessaloniciens: c'est-à-dire l'idée d'une puissance de délai qui retarde la fin du temps. Dans Le Nomos de la terre - qui est sorti la même année que la critique sur le livre de Löwith - l'idée du katechon synthétise la conception schmittienne de l'empire chrétien:

Je ne crois pas que la foi chrétienne puisse avoir en général une image de l'histoire différente de celle du katechon. La foi en une force freinante en mesure de retenir la fin du monde jette les seuls ponts qui, à partir de la paralysie eschatologique de chaque événement, conduisent à une grandiose puissance historique comme celle de l'empire chrétien des rois germaniques. ${ }^{17}$

La figure du katechon, sur laquelle Schmitt se concentre de façon explicite au début des années 1950, se révèle tout à fait cohérente avec la définition théologico-politique de la souveraineté nationale qu'il a élaborée dès le début des années $1920^{18}$. En face de la vision eschatologico-apocalyptique du temps, il est attiré par la prégnance d'une semblable perspective, mais il sent aussi le besoin d'individuer «une force qui retient la fin». De la même façon, il perçoit la portée révolutionnaire de la contingence, à laquelle le système juridique

15. Ibid., p. 928.

16. Ibid., p. 929.

17. C. Schmitt, Der Nomos der Erde im Völkerrecht des Jus Publicum Europaeum, Berlin, Duncker \& Humblot, 1997, p. 29; Le nomos de la terre dans le droit des gens du Jus Publicum Europaeum, éd. Peter Haggenmacher, trad. Lilyane Deroche-Gurcel, Paris, Presses Universitaires de France, 2001, p. 64.

18. Voir C. Schmitt, Politische Theologie, Munich \& Leipzig, Duncker \& Humblot, 1922; Théologie politique, trad. Jean-Louis Schlegel, Paris, Gallimard, 1988. 
est exposé grâce à la décision souveraine sur «l'état d'exception». Toutefois, son intérêt est toujours la protection de la légitimité de la loi, qui est inscrite dans la logique freinante du droit. Cette position le porte à défendre la nécessité d'un pouvoir katechontique, malgré la prise de conscience que les temps ont changé et que de nouvelles perspectives sont apparues sur la scène mondiale de l'histoire.

\section{LE NOMOS DANS L'UNITÉ DU MONDE}

Quelques années après la publication du Nomos de la terre et de la critique du livre de Löwith, Schmitt entretient un échange épistolaire avec Alexandre Kojève ${ }^{19}$, le plus important théoricien de la fin «apocalyptique» de l'histoire, élaborée à partir d'une lecture de la Phénoménologie de l'esprit de Hegel, qui a marqué une époque ${ }^{20}$. La question qui occupe Kojève et Schmitt dans cette correspondance est celle qui concerne le destin historique du «politique» à l'époque de l'unité du monde, où le marché global domine sans conteste, à travers la standardisation et l'unification des différents points de la planète. Tous deux reconnaissent la crise de l'État national moderne et de sa structure juridique. Mais pour Schmitt le problème du nomos, de la lutte politique autour du nomos et du pouvoir qui se fonde sur lui, existe aussi à l'époque de la politique globale. Il «ne considère toujours pas notre planète terre, aussi petite puisse-t-elle être devenue, comme une unité de planification (Planungs-Einheit)» (lettre du 7 juin 1955). Selon lui, en «opposition à l'unité du monde», «une pluralité» est encore possible, «et, par là, une hostilité douée de sens (sinnvolle Feindschaft)», qui «fonde l'aptitude à faire l'histoire» contre «la supposition - qu'il impute à Kojève - que le cercle du temps s'est déjà bouclé» (ibid.).

Pour Schmitt, il est vrai qu'à l'époque du «grand espace» la question du pouvoir subit de profondes transformations: au lieu de la politique mondiale s'instaure une espèce de «police mondiale»

19. Voir Der Briefwechsel Kojève-Schmitt, in Schmittiana. Beiträge zu Leben und Werk Carl Schmitts, éd. Piet Tommissen, Bd. 6, Berlin, Duncker \& Humblot, 1998, p. 100-124.

20. Voir Alexandre Kojève, Introduction à la lecture de Hegel, Paris, Gallimard, 1979. 
et, à rebours des guerres connues jusqu'alors, se crée une unique «guerre civile mondiale». À partir de cette prise de conscience, dans la lettre du 16 mai 1955, Kojève se demande et demande à Schmitt: «existe-t-il encore des États (Staaten) au sens véritable du mot, c'est-à-dire des gouvernements (Regierungen) qui soient autre chose que des administrations (Verwaltungen), et une politique (= guerre) qui signifie davantage qu'une police?». Une fois devenue police planétaire, la politique ne semble être autre chose qu'une simple administration.

Même Schmitt est conscient de cette question. Dans un essai du début des années 1950, qu'il a envoyé à Kojève (comme on peut le lire dans la correspondance), il admet que, aujourd'hui, «le niveau de vie est de plus en plus haut, la division devient de plus en plus facile, de plus en plus inoffensive», si bien que la lutte pour l'appropriation - sur laquelle, selon lui, se fonde le sens du pouvoir politique dans l'histoire - apparaît finalement «irrationnelle du point de vue économique et donc insensée $»^{21}$. Dans un autre texte, publié à la même époque, Schmitt écrit: «En d'autres mots, l'humanité aurait enfin trouvé sa formule, de même que l'abeille a trouvé sa ruche. Les choses se gouvernent par elles-mêmes; l'humanité se rencontre elle-même; la migration dans le désert de l'aliénation est à sa fin $»^{22}$. Les mots écrits par Schmitt dans cet essai rappellent de façon impressionnante ceux formulés par Kojève en 1968, dans la note additionnelle à la seconde édition de l'Introduction à la lecture de Hegel, dans laquelle la fin de l'histoire, parallèle à l'accomplissement de l'État universel et homogène, est décrite dans les termes d'un complet retour de l'homme à l'animalité, lorsque le logos devient semblable au «"langage" des abeilles »"23.

La correspondance entre Schmitt et Kojève met en relief une indubitable lucidité des deux positions. Mais les faits les plus récents démontrent aussi leur faiblesse. Dans les dernières années, à un «niveau de vie de plus en plus haut» n'a pas correspondu une

21. C. Schmitt, «Nehmen / Teilen / Weiden. Ein Versuch, die Grundfragen jeder Sozial- und Wirtschaftsordnung vom Nomos her richtig zu stellen», Gemeinschaft und Politik, 1, 3, 1953, p. 18-27.

22. C. Schmitt, «Nomos Nahme Name», Der Beständige Aufbruch. Festschrift für Erich Przywara S.J., éd. Siegfried Behn, Nuremberg, Verlag Glock, 1959, p. 92-105.

23. A. Kojève, Introduction à la lecture de Hegel, p. 436. 
«distribution de plus en plus facile, de plus en plus inoffensive», comme l'avait soutenu Schmitt, d'accord avec Kojève. En revanche, une implosion de la classe moyenne a touché de façon massive les pays occidentaux, jusqu'à en arriver à sa quasi-disparition. L'écart entre pauvreté et richesse a augmenté de façon vertigineuse, devenant l'un des aspects les plus importants de l'actuelle crise de l'économie mondiale. De nouvelles analyses du phénomène et de nouvelles réflexions sur le destin de la politique dans la crise du marché global sont désormais nécessaires. Mais avant de tenter une enquête en ce sens, il vaut la peine de se demander en quel sens il est possible d'individuer, dans la perspective de Schmitt, une tentative de défense de l'histoire, même à l'époque de l'administration planétaire, lorsque chaque événement semble avoir complètement perdu son sens et que le langage humain apparaît toujours plus semblable à celui des abeilles. À cet égard, on ne peut pas ignorer son interprétation de l'histoire chrétienne.

Dans la critique du livre de Löwith, il réfléchit sur le sens de l'histoire chrétienne et affirme que cette dernière consiste en un «événement historique d'une unicité infinie, inappropriable, inoccupable» et que c'est précisément ce qui fait que, pour le chrétien, le comportement historique est marqué par «un manque, une privation et une impuissance qui fortifie $»^{24}$. Ainsi, avec une image qui reprend en même temps qu'elle renverse celle de l'ange de l'histoire des thèses benjaminiennes ${ }^{25}$, Schmitt écrit que «le Christ regarde en arrière vers des événements pleinement advenus pour y trouver un parangon et une incarnation dans la contemplation desquels le sens obscur de notre histoire continue de croître $»^{26}$. Il localise donc une figure pour ce regard jeté a posteriori sur l'histoire, celle de l'Épiméthée chrétien, décrite par le poète allemand Konrad Weiss. Épiméthée doit se confronter avec le sens d'une action marquée par le «manque, la privation et une impuissance qui fortifie». Tout comme l'homme contemporain, Épiméthée est responsable d'une action qui, de fait, n'a aucun pouvoir pour déterminer l'histoire, qui ne décide rien et ne fait que réaliser ce qui a été prédéterminé (il ne faut pas oublier que c'est précisément sur ces bases que Löwith accuse Schmitt

24. C. Schmitt, «Drei Stufen historischer Sinngebung», p. 930.

25. Voir Giorgio Agamben, «Introduzione», in C. Schmitt, Un giurista davanti a se stesso. Saggi e interviste, éd. G. Agamben, Vicenza, Neri Pozza, 2005, p. 15.

26. C. Schmitt, «Drei Stufen historischer Sinngebung», p. 930. 
d'occasionnalisme ${ }^{27}$ ). À l'instar de la figure chrétienne du katechon, Épiméthée inaugure lui aussi un temps dans lequel rien ne peut vraiment advenir. En enrayant l'événement eschatologique, il en retarde la venue dans un devenir historique indéfini, un temps sans le temps, tout à fait semblable à la «fin de l'histoire» souhaitée par Kojève; mais surtout extrêmement proche de la condition d'hommes qui, comme les fonctionnaires nazis ${ }^{28}$, décident seulement de ce qui a déjà été programmé.

\section{Messianisme et aPocalyptiQue}

En 1978, Schmitt rencontre à Plettenberg Jacob Taubes, rabbin qui n'en exerça jamais la charge, mais surtout l'auteur, entre autres, d'une interprétation théologico-politique de l'Épître aux Romains de Paul ${ }^{29}$. Dans une longue lettre adressée au juriste allemand et rédigée après leur rencontre, Taubes le remercie pour la «patience» et la «franchise» avec laquelle il lui «a parlé aussi des erreurs commises dans sa longue vie de légiste» et écrit:

Justement, en tant qu' «Archijuif» $\left(\right.$ Erzjude $^{30}$, j' ai appris à hésiter avant de jeter la pierre. Parce que, dans toute cette horreur sans nom, nous sommes restés à l'abri d'une chose. Nous n'avions aucun choix: Hitler nous avait élus comme l'ennemi absolu. Or là où il n'y a aucun choix, il n'y a non plus aucun jugement, et surtout pas sur autrui. Cela ne signifie pas que je ne sois pas hanté par le besoin de comprendre ce qui s'est «véritablement» passé (pas du tout dans le sens historique, mais bien plutôt dans celui eschatologique de l'entrée en crise) - là où s'est opéré l'aiguillage vers les catastrophes (la nôtre et la vôtre). ${ }^{31}$

27. Voir K. Löwith, «Der okkasionelle Dezisionismus von C. Schmitt», Sämliche Schriften, Stuttgart, Metzler, 1984, B. 8.

28. Cf. G. Agamben, «Introduzione», p. 17.

29. Cf. Jacob Taubes, Die politische Theologie des Paulus, éd. Aleida et Jan Assmann, Munich, Fink, 1993; La théologie politique de Paul. Schmitt, Benjamin, Nietzsche, Freud, trad. Mira Köller et Dominique Séglard, Paris, Éditions du Seuil, 1999. À propos de cet auteur voir aussi E. Stimilli, Jacob Taubes. Sovranità e tempo messianico, Brescia, Morcelliana, 2004.

30. Erzjude est un mot forgé par Martin Buber (pour parler de Karl Kraus) sur le modèle de Erzbischof (archevêque), Erz- étant la germanisation du grec archè.

31. J. Taubes, Ad Carl Schmitt. Gegenstrebige Fügung, Berlin, Merve, 1987, p. 39; En divergent accord. À propos de Carl Schmitt, trad. Philippe Ivernel, préf. d'E. Stimilli, Paris, Payot \& Rivages, 2003, p. 67-68. Une traduction de la lettre du 18 septembre 1979 à Carl Schmitt figure également en annexe de $L a$ théologie politique de Paul, p. 175-179. (La traduction ici adoptée modifie les 
Le problème eschatologico-apocalyptique de l' «état d'urgence», sur lequel se dresse la théorie schmittienne de la souveraineté, oblige à une confrontation avec l'accomplissement des événements dans la «décision», qui implique le sens de l'action historique au moment du plus grand danger. Taubes en vient, sur ce point, à se confronter directement avec Schmitt et décide de le rencontrer dans sa maison de Plettenberg. De leur rencontre émerge un intérêt commun pour l'idée chrétienne de la fin apocalyptique des événements historiques. "Carl Schmitt, écrit-il, pense en termes d'apocalypse, mais d'en haut, à partir des puissances », tandis que Taubes pense «d'en bas». Ce qui les réunit, en revanche, c'est l'«expérience du temps et de l'histoire comme délai (Frist), comme dernier délai (Galgenfrist). C'est aussi originairement, ajoute Taubes, une expérience chrétienne de l'histoire $»^{32}$. En suivant cette piste, Taubes en arrive de Schmitt à Benjamin. Un chemin difficile à travers lequel il ne peut échapper à la confrontation avec deux des plus importants représentants de la culture judéo-allemande d'après-guerre, amis de Benjamin et connus comme les premiers et les plus influents interprètes et diffuseurs de son œuvre: Théodore W. Adorno et Gershom Scholem.

Vers 1969, Taubes commence à parler de la lettre que Benjamin a envoyée à Schmitt en 1930, un document qui ne fait pas partie de la correspondance benjaminienne publiée par Adorno et Scholem en 1966. Cette lettre constitue un des points les plus douloureux de sa relation avec Scholem, auquel il n'a pas manqué de faire remarquer que Schmitt l'a rendue publique dix ans avant la publication de la correspondance benjaminienne ${ }^{33}$. Contre l'interprétation de la pensée de Benjamin proposée par Scholem, et surtout contre sa propre idée de messianisme, Taubes entreprend une lecture comparée de Schmitt et de Benjamin, qu'il n'a jamais interrompue jusqu'à la finn ${ }^{34}$. À cet

deux traductions existantes). À propos de cette lettre et de tous les documents relatifs à la relation entre Taubes et Schmitt, voir Jacob Taubes - Carl Schmitt. Briefwechsel mit Materialien, éd. Herbert Kopp-Oberstebrink, Thorsten Palzhoff et Martin Treml, Munich, Fink, 2011.

32. Ibid., p. 22 ; tr. fr., p. 45.

33. Voir Jacob Taubes. Der Preis des Messianismus. Briefe von Jacob Taubes an Gershom Scholem und andere Materialen, éd. E. Stimilli, Würzburg, Königshausen und Neumann, 2006, p. 116, 117 et 119.

34. À ce propos, voir, Jacob Taubes - Carl Schmitt. Briefwechsel, p. 211-213, et E. Stimilli, «Der Messianismus als politisches Problem», Jacob Taubes. Der Preis des Messianismus, p. 131-179. 
égard, les notes publiées du séminaire sur les Thèses sur le concept d'histoire de Benjamin, que Taubes a tenu à la Freie Universität de Berlin au cours du semestre d'hiver 1984-1985, constituent un document particulièrement important ${ }^{35}$. Dans sa lecture, Taubes met en évidence un «concept de théologie qui s'accorde avec l'immanence radicale du matérialisme historique $»^{36}$, afin de définir un nouveau concept d'histoire. En particulier, commentant la troisième thèse ${ }^{37}$, il affirme:

Ce n'est qu'à la fin que tout ce qui appartient au passé sera visible et transparent - à vrai dire, ici Benjamin tourne en rond. Le Jugement dernier n'est pas le spectacle à la fin; il est au contraire possible dans chaque remémoration pleine. Dans cette perspective - à partir d'elle -, le refus d'un état final est - à vrai dire: devient -, compréhensible: la rédemption est possible à chaque instant. Le Jugement dernier est incorporé au temps, qui peut rompre. Ici se trouve le lieu de la critique par Benjamin de l'image théologique comme de l'idée de matérialisme historique; [pour l'une comme pour l'autre] en effet, le Jugement dernier est le spectacle de la fin. Il en va autrement chez Benjamin: inclusion de l'idée de fin dans le temps lui-même. Ce qui veut dire : irruption ponctuelle, éclair. Aucun jour n'est exclu. La forme apocalyptique est donc investie dans un temps présentiste. ${ }^{38}$

Chez Benjamin, la sortie d'une configuration apocalyptique de l'histoire qui conçoit le «jour du Jugement» comme «spectacle de la fin» est portée à son terme. Pour lui, «la rédemption est possible à chaque instant» et il introduit «l'idée de la fin au sein du temps lui-même». Taubes adhère à cette forme de libération, en se libérant lui-même des antinomies qui peuvent trouver leur origine dans une conception ambiguë de l'apocalypse ${ }^{39}$. À travers Benjamin, Taubes explore ainsi un passage de l'apocalyptique au messianisme. «Benjamin ne défend pas un futurologisme messianique, mais un

35. J. Taubes, «Walter Benjamin: Geschichtsphilosophische Thesen», Jacob Taubes. Der Preis des Messianismus, p. 67-92.

36. Ibid., p. 71.

37. Voir Walter Benjamin, «Über den Begriff der Geschichte», Gesammelte Schriften, Bd. I/2, Francfort-sur-le-Main, Suhrkamp, 1974, p. 691-704, p. 694; «Sur le concept d'histoire», CEuvres, tome 3, trad. Maurice de Gandillac, Rainer Rochlitz et Pierre Rusch, Paris, Gallimard, p. 427-443, p. 429

38. J. Taubes, «Walter Benjamin: Geschichtsphilosophische Thesen», p. 74.

39. À ce propos, voir J. Taubes, Abendländische Eschatologie [1947], Munich, Matthes \& Seit Verlag, 1991; Eschatologie occidentale, trad. Raphaël Lellouche et Michel Pennetier, Paris, Éditions de l'Éclat, 2009; et Abendländische Eschatologie. Ad Jacob Taubes, éd. Richard Faber, Eveline Goodman-Thau et Thomas Macho, Würzburg, Königshausen \& Neumann, 2001. 
actualisme messianique $»^{40}$. Cela veut dire qu'il transfère au centre l'élément critique et fait de la crise et du "péril» l'épicentre d'une expérience authentique de l'histoire. Dans une des dernières pages des notes de conférence, il affirme: «La communauté messianique n'est pas sans histoire, tout ce qui est passé est sans cesse susceptible de sourdre dans un maintenant, est donné dans l'état d'exception permanent $\rangle^{41}$. Taubes se réfère ici, évidemment, à la huitième thèse sur le concept d'histoire ${ }^{42}$, dans laquelle Benjamin décrit, de façon plus ou moins explicite, le règne messianique à la lumière de la définition schmittienne de la souverainetée ${ }^{43}$ et semble vouloir établir un rapport entre la venue du Messie et le concept clé du système juridique. Percevant la force et la contingence de la théorie schmittienne de la souveraineté, c'est comme s'il voulait pousser cette perspective jusqu'à ses dernières conséquences, en démontrant comment le fondement caché de la Loi est celui dans lequel le droit est dans un état de suspension absolue, une crise réelle: un «état d'exception effectif». Tandis que Schmitt a tendance à dire qu'il n'y a pas de «dehors» de la Loi, au point que, pour lui, souveraineté et légalité en viennent à signifier la même chose, l'intérêt de Benjamin est, en revanche, de saisir, dans le souverain, un excès de pouvoir juridique. On peut dire que ceci est aussi le cœur de la réflexion antinomique de Paul de Tarse, que Taubes a exposée en termes théologico-politiques ${ }^{44}$.

Le fait qu'un philosophe juif des religions, petit-fils et fils de rabbin, qui aurait pu être rabbin lui-même, ait rencontré celui qu'il désigne comme «le plus grand constitutionnaliste de notre temps», qui était aussi profondément catholique de culture et le théoricien du régime nazi, qu'ils aient discuté, entre autres choses, de l'Épître aux Romains est presque incroyable ${ }^{45}$. Mais ce qui est encore plus déconcertant est que, sur les pas de Benjamin, Taubes semble trouver en Schmitt un interlocuteur encore plus anticonformiste que Scholem pour une lecture politique de Paul, même si son interprétation est en contradiction avec la position du dogme

40. J. Taubes, «Walter Benjamin: Geschichtsphilosophische Thesen», p. 75.

41. Ibid., p. 90.

42. Voir W. Benjamin, «Über den Begriff der Geschichte», p. 697.

43. Voir C. Schmitt, Politische Theologie, p. 11.

44. Voir J. Taubes, Die politische Theologie des Paulus.

45. Voir ibid., p. 10-11. 
chrétien, puisqu'il cherche dans les racines juives du messianisme paulinien le sens d'une vie post Christum $^{46}$. Schmitt se révèle ainsi être son véritable adversaire. Les deux positions restent inchangées. À la fin de sa vie, Taubes définit cette relation comme un «divergent accord»-l'un en tant qu' «apocalyptique de la contre-révolution», l'autre en tant qu' «apocalyptique de la révolution $»^{47}$.

Il ne faut pas, sur ces prémisses, oublier que la réflexion de Taubes a été redécouverte et interprétée de diverses manières à notre époque, précisément au moment où le déclin du pouvoir juridique des États nationaux est désormais exposé aux yeux de tous, et, à la lumière de la suprématie du marché global, l'effondrement des démocraties occidentales est en train de devenir un phénomène de plus en plus problématique. De nouvelles «apocalypses» sont annoncées sans que l'on puisse entrevoir une voie pour renverser les puissances du monde. Je crois qu'une réflexion sur ce à quoi nous sommes en train d'assister ne peut se dispenser de prendre sérieusement en considération la force "antinomique» qui est intrinsèque au néolibéralisme et au type de gouvernement économique qu'ont favorisé les politiques néolibérales durant ces dernières années. Même dans ce cas, il peut être assez utile de repenser le rôle de la religion chrétienne dans le développement de la modernité.

\section{Pouvoir PaSTORAL, APOCALYPTIQUE ET GOUVERNEMENTALITÉ}

Une des plus importantes contributions dans ce domaine a été donnée par Michel Foucault qui, dans ses derniers cours au Collège de France, a ouvert un chantier en se mettant à réfléchir sur le pouvoir économique en tant que «pouvoir gouvernemental», en tentant de comprendre la force et la nouveauté du néolibéralisme, dont il avait déjà saisi, depuis la fin des années 1970, la capacité à s'affirmer à l'échelle globale ${ }^{48}$. Le but de ces réflexions est de définir, d'une manière spécifique, la nouvelle technique de gouvernement dont

46. Voir E. Stimilli, «Der Messianismus als politisches Problem», p. 155-179.

47. Voir J. Taubes, Ad Carl Schmitt, p. 20; tr. fr., p. 43.

48. Voir Michel Foucault, Sécurité, territoire, population. Cours au Collège de France. 1977-1978, Paris, Gallimard - Seuil - EHESS, 2004, et Naissance de la biopolitique. Cours au Collège de France, 1978-1979, Paris, Gallimard - Seuil - EHESS, 2004. 
le néolibéralisme s'est fait le chantre. Selon lui, le prototype du pouvoir gouvernemental est le «pouvoir pastoral chrétien $»^{49}$.

Dans son cours de 1978, tout en rappelant l'intersection qu'il y a entre le pouvoir pastoral et le pouvoir politique, Foucault tient à souligner l'hétérogénéité de ces deux modes de gouvernement, qu'il perçoit comme «un des traits de l'Occident» chrétien ${ }^{50}$. L'analyse du pastorat est ainsi menée par Foucault dans l'intention de distinguer radicalement le pouvoir gouvernemental et le pouvoir souverain. Dans ce sens, il montre l'hétérogénéité du pouvoir gouvernemental par rapport à la logique théologico-politique de la souveraineté. Dès lors, la généalogie foucaldienne de la gouvernementalité moderne - l'analyse du pastorat chrétien - se présente comme une archéologie de l'idée de théologie politique, en même temps que comme sa déconstruction radicale.

Ainsi, Foucault exclut de son analyse du pastorat les questions liées au domaine de l'apocalyptique et de l'eschatologie. Dans le cours de 1978, par exemple, une absence saute aux yeux: l'omission du thème de la sécularisation, qui - comme nous l'avons vu avec Löwith - présuppose la vision chrétienne de l'histoire comme histoire du salut et la définition eschatologique qui s'ensuit de l'Église comme institution du salut. Le salut, comme opérateur politique, n'importe guère en tant qu'élément sécularisé et immanent dans le processus téléologique de l'histoire de l'Église. Son intérêt réside plutôt, selon Foucault, dans les effets qu'il produit sur la technique même du pouvoir pastoral. C'est à travers cette forme de pouvoir que le christianisme a su gouverner les hommes omnes et singulatim, jusque dans leur vie la plus intime et quotidienne ${ }^{51}$. En ce sens, Foucault ne fait pas du salut un principe interprétatif de l'histoire du christianisme et de la modernité occidentale comprise comme processus unique, comme développement universel soumis au processus de sécularisation.

En tentant d'expliquer le passage, selon lui pas tout à fait clair chez Foucault, «du pastorat ecclésiastique au gouvernement

49. Michel Foucault, Sécurité, territoire, population, p. 151.

50. Ibid., p. 158.

51. Voir M. Foucault, «Omnes et singulatim. Toward a Criticism of Political Reason, The Tanner Lectures on Human Values, éd. Sterling Mc Murrin, Salt Lake City, University of Utha Press, 1981, p. 223-254; et Dits et écrits II, 1976-1988, Paris, Gallimard, 2001, p. 953-980. 
politique», Giorgio Agamben a récemment récupéré la vision chrétienne de l'histoire du salut comme perspective herméneutique du pouvoir gouvernemental et a souligné l'absence, dans le cours de Foucault de 1978, de toute référence au concept de providence comme gubernatio divine du monde distincte du règne divin ${ }^{52}$. Ce faisant, il réduit l'analyse foucaldienne du pastorat au discours sur la sécularisation, avec lequel Foucault entend précisément prendre ses distances.

Foucault s'intéresse au christianisme parce qu'il y découvre la capacité d'inventer une nouvelle technique de pouvoir, le pouvoir gouvernemental, fondé sur une forme d'obéissance particulière, qu'il définit comme «obéissance pure $»^{53}$. Il s'agit d'une obéissance qui n'est pas engendrée à partir d'une soumission à la loi, mais plutôt à partir d'un lien de confiance pour celui qui est en mesure de diriger les troubles de l'âme sur la base d'un libre «gouvernement de soi». Dans l'analyse que fait Foucault du pastorat chrétien, la force antinomique du christianisme - qui émerge aussi dans la lecture du messianisme paulinien proposée par Taubes - est interprétée en termes de technique de gouvernement, qui se révèle être la matrice de la gouvernementalité libérale ${ }^{54}$. Ainsi, Foucault considère le libéralisme comme un stratagème de la religion chrétienne elle-même.

C'est dans ce sens que la dimension politique n'apparaît pas, pour Foucault, comme une donnée, mais plutôt comme l'effet d'une série compliquée de constructions de gouvernement, dont le caractère à l'origine non politique n'autorise pas à les considérer seulement comme une cause efficiente. Le gouvernement est plutôt le moyen à travers lequel la politique se déploie historiquement dans les formes instituées du pouvoir. Même l'État se révèle ainsi comme une «péripétie du gouvernement», une forme de gouvernement parmi d'autres et non l'essence même du pouvoir ${ }^{55}$.

52. Voir G. Agamben, Il Regno e la Gloria. Per una genealogia teologica dell'economia e del governo, Milan, Neri Pozza, 2007, p. 125-128; Le Règne et la Gloire. Pour une généalogie théologique de l'économie et du gouvernement, trad. Joël Gayraud et Martin Rueff, Paris, Le Seuil, 2008, p. 173-177

53. M. Foucault, Sécurité, territoire, population, p. 177.

54. À propos d'une analyse du christianisme dans cette direction, avec une attention particulière au problème de la faute en tant que dette, je renvoie à mes livres: E. Stimilli, Il debito del vivente. Ascesi e capitalismo, Macerata, Quodlibet, 2011 et Debito e colpa, Ediesse, Roma, 2015.

55. M. Foucault, Sécurité, territoire, population, p. 282. 
Sa solidité dépend précisément du fait que la souveraineté de l'État est le résultat de pratiques gouvernementales complexes. Ce n'est que dans la configuration économique que la politique trouve sa version la plus radicale, en particulier dans le néolibéralisme, qui, par son ingouvernabilité intrinsèque, permet de mettre en évidence la liberté comme technique de gouvernement.

Au cœur du travail de Foucault - qui consiste essentiellement en un sondage des différentes formes de connexion entre l'ingouvernable et ce qui est gouverné dans les véritables figures historiques du pouvoir politique - il y a la conviction que le gouvernement est proprement le «gouvernement des hommes", en ce sens que les êtres humains sont des êtres vivants qui se gouvernent eux-mêmes et, en même temps, sont une matière qui doit être gouvernée. Dans cet horizon, le champ individuel et le champ social sont si intimement liés que, dans le même temps, ils sont le fruit d'un «processus de subjectivisation", dans lesquels l'élément ingouvernable est continuellement et nécessairement imbriqué dans une activité gouvernementale. $\mathrm{Ce}$ n'est donc pas un hasard si Foucault consacre une grande partie des dernières années de sa vie à l'étude des techniques anciennes de constitution du sujet ${ }^{56}$, repérant dans la religion chrétienne un tournant décisif pour une histoire de la gouvernementalité, l'origine d'une forme inédite de constitution du sujet comme «champ d'obéissance généralisé ${ }^{57}$. Mais la constitution du pouvoir, pour Foucault, en tant que rapport de forces, prévoit aussi de temps en temps des points de résistance, qui seuls peuvent modifier, de l'intérieur, ces différentes réalisations historiques.

C'est sur cette voie que, dans son cours de 1978, Foucault affronte la question de la «foi eschatologique» dans le cadre des luttes antipastorales, comme manifestations historiques de certaines formes de résistance au pouvoir gouvernemental. Avec le mot «eschatologie», Foucault entend ici n'importe quelle idée de type «chiliastique» fondée sur le modèle apocalyptique hérité de la tradition juive, c'est-à-dire celui qui prévoit l'avènement d'un millenium durant lequel l'humanité, complètement purifiée, pourra jouir d'une

56. Voir en particulier L'herméneutique du sujet. Cours au Collège de France, 1981-1982, Paris, Gallimard - Seuil - EHESS, 2001, et Le gouvernement de soi et des autres. Cours au Collège de France, 1982-1983, Paris, Gallimard - Seuil EHESS, 2008.

57. M. Foucault, Sécurité, territoire, population, p. 182. 
vie parfaitement heureuse. La vision apocalyptique représente, par conséquent, une contestation de l'ordre social, politique et religieux institué. C'est dans ce sens que Foucault affirme que «le christianisme, dans son organisation pastorale réelle [...] n'est pas une religion de l'eschatologie $»^{58}$. On doit insister sur le fait que l'aspect révolutionnaire de cette perspective eschatologico-apocalyptique ne doit pas être individué au sein d'un développement évolutif unique qui serait ainsi complètement subverti. Ce qui est révolutionnaire, c'est plutôt la potentialité intrinsèque de tout rapport de pouvoir, dans un sens qui la rapproche de «l'actualité messianique» élaborée par Benjamin et mise en lumière par Taubes.

De toute façon, dans sa confrontation avec le christianisme, l'intérêt de Foucault - comme celui de Nietzsche - n'est pas du tout de parvenir à un discours destiné à identifier une «origine» ou une «essence». Son intention est plutôt de discerner «une autre histoire du christianisme $»^{59}$, de mettre au jour «l'invention» réalisée par le christianisme: la nouvelle technique de gouvernement fondée sur une forme paradoxale et antinomique de «libre obéissance», qui n'est pas engendrée par la force de la loi, mais plutôt à travers son individualisation. Tout comme Nietzsche, Foucault refuse la recherche d'une origine «pure» du christianisme, qui serait par la suite trahie et sécularisée par sa propre histoire. Non seulement parce que l' "origine» (Ursprung), d'une certaine façon, n'existe pas ou n'est pas clairement identifiable comme donnée initiale. Mais surtout parce que ce qui l'intéresse le plus dans le christianisme - et dans sa «force politique»- est le fait d'avoir su créer les conditions de cette nouvelle façon de se gouverner soi-même et de gouverner les autres: une forme de dépendance à travers la liberté, au sein de laquelle il est peut-être encore possible de trouver des points de résistance.

<elstimilli@gmail.com>

58. Ibid., p. 218.

59. Voir Michel Senellart, «Michel Foucault: une autre histoire du christianisme?», Bulletin du centre d'études médiévales d'Auxerre, 7, 2013: http://cem. revues.org/12872, ainsi que Philippe Chevallier, Foucault et le christianisme, Lyon, ENS Éditions, 2011 et "Étudier l'Église comme "gouvernementalité" ", Bulletin du centre d'études médiévales d'Auxerre, 7, 2013: http://cem.revues. org/12874. 